\title{
Surveillance summary of hospitalized pediatric enterovirus D68 cases in Canada, September 2014
}

\author{
Edwin $\mathrm{JJ}^{1^{*}}$, Reyes Domingo $\mathrm{F}^{2}$, Booth $\mathrm{TF}^{3}$, Mersereau $\mathrm{T}^{2}$, Skowronski $\mathrm{DM}^{4}$, Chambers $\mathrm{C}^{4}$, Simmonds $\mathrm{K}^{5}$, \\ Scott $A N^{5}$, Winter $A L^{6}$, Peci $A^{6}$, Gubbay $J^{6}$, Drews $S J^{7}$, Krajden $M^{4}$, Karnauchow $\mathrm{T}^{8}$, Smieja $\mathbf{M}^{9}$, \\ Rempel $S^{1}$, Murti $M^{10}$, Pollock $S^{11}$, Gustafson $R^{12}$, Hoyano $D^{13}$, Allison $S^{14}$, Fathima $S^{7}$, Pabbaraju $K^{7}$, Wong \\ $S^{7}$, Tellier $R^{7}$, Tipples $G^{7}$, Gad $R^{15}$, Mukhi $S N^{3}$, Jafari $Y^{16}$, Grudeski $E^{3}$, McDermid $A^{3}$, Wong $T^{2}$
}

${ }^{1}$ Centre for Public Health Infrastructure, Public Health Agency of Canada, Ottawa, ON

${ }^{2}$ Centre for Immunization and Respiratory Infectious Diseases, Public Health Agency of Canada, Ottawa, ON

${ }^{3}$ National Microbiology Laboratory, Public Health Agency of Canada, Winnipeg, MB

${ }^{4}$ British Columbia Centre for Disease Control, Vancouver, BC

${ }^{5}$ Alberta Health, Edmonton, $\mathrm{AB}$

${ }^{6}$ Public Health Ontario, Toronto, ON

${ }^{7}$ Alberta Provincial Laboratory for Public Health, Edmonton and Calgary, AB

${ }^{8}$ Children's Hospital of Eastern Ontario, Ottawa, ON

${ }^{9}$ Hamilton Regional Laboratory Medicine Program, Hamilton, ON

${ }^{10}$ Fraser Health Authority, Surrey, BC

${ }^{11}$ Interior Health Authority, Kelowna, BC

${ }^{12}$ Vancouver Coastal Health Authority, Vancouver, BC

${ }^{13}$ Vancouver Island Health Authority, Victoria, BC

${ }^{14}$ Northern Health Authority, Prince George, BC

${ }^{15} \mathrm{New}$ Brunswick Department of Health, Fredericton, NB

${ }^{16}$ Northwest Territories Health and Social Services, Yellowknife, NT

${ }^{*}$ Corresponding author: jonathan.edwin@phac-aspc.gc.ca

\section{Abstract}

Background: Enterovirus D68 (EV-D68) has been detected infrequently and has not been associated with severe disease in Canada. In the early fall of 2014, following an unusual case increase in the United States, clusters of EV-D68 among children and some adults manifesting severe symptoms were reported in Canada.

Objective: To provide an initial epidemiological summary of pediatric cases hospitalized with EV-D68 in Canada.

Methods: A time-limited surveillance pilot was conducted collecting information on pediatric cases (less than 18 years of age) hospitalized with EV-D68 between September 1 and 30, 2014.

Results: In total, 268 cases were reported from Ontario $(n=210)$, Alberta $(n=45)$, and British Columbia $(n=13)$. Of the 268 reported cases, $64.9 \%(n=174)$ were male; the sex difference was statistically significant $(p<0.01)$. Age was reported for 255 cases, with a mean age for males of 5.4 years and for females of 5.3 years. For cases with data available, $6.8 \%$ (18/266) were admitted to an intensive care unit. Of those where clinical illness was recorded, respiratory illness alone was present in $98.3 \%$ (227/231), neurologic illness alone was present in $0.4 \%$ $(n=1)$, and both illnesses were present in $0.9 \%$ of cases $(n=2)$; cases with neither respiratory nor neurologic illness were rare $(n=1)$. Of the 90 cases with additional clinical information available, $43.3 \%$ were reported as having asthma. No deaths were reported among the 268 cases.

Conclusion: The EV-D68 outbreak in Canada in September 2014 represents the beginning of a novel outbreak associated with severe illness in children. These findings provide the first epidemiological summary of severe cases of EV-D68 as an emergent respiratory pathogen in Canada. The continued investigation of this pathogen is necessary to build on these results and capture the full spectrum of associated illness. 


\section{Introduction}

Enterovirus D68 or EV-D68 (genus enterovirus) is a non-polio enterovirus of the Picornaviridae family (1). It was first discovered in 1962 after it was isolated from four children ill with pneumonia and bronchiolitis in California (2). Historically, detections of EV-D68 have been infrequent (3). From 1970 to 2005, the United States National Enterovirus Surveillance System identified EV-D68 in only 26 of 49,637 (0.1\%) laboratory-typed enterovirus specimens identified during this 36-year period (4). In Canada, a total of 82 cases of EV-D68 were identified by the National Microbiology Laboratory from 1999 to 2013 (5).

Since 2008, several outbreaks of EV-D68 have been reported internationally, including in the Philippines (2008-2009) (6), Japan $(2010)(7,8)$, the Netherlands $(2010)(9,10)$, and the United States $(2009-2010)(11,12)$. Reported illness ranged from mild symptoms associated with respiratory illness (fever, runny nose, sneezing, coughing, and body and muscle aches) to severe disease, primarily pneumonia and bronchiolitis, requiring intensive care (12). Infections were reported in both children and adults; however, children aged less than 1 month to 4 years old were most commonly affected. Children with underlying respiratory disease (e.g., asthma, wheezing, and bronchiolitis) and immunocompromised adults have been reported to experience more severe disease $(13,14)$. The epidemiology and full spectrum of disease caused by EV-D68 remains unclear $(12,13)$, primarily due to its infrequent detection. However, its recent emergence as a pathogen with outbreak potential associated with severe illness (7-12) provides impetus for investigation.

\section{Event description}

In mid-August 2014, the U.S. Centers for Disease Control and Prevention (CDC) was notified of increases in severe respiratory illness in children in Kansas City and Chicago. Further testing identified EV-D68 as the predominant etiologic agent detected (13). From mid-August to December 11, 2014, the outbreak had spread across the country resulting in 1,149 laboratory-confirmed EV-D68 cases and 12 deaths reported from 48 states and the District of Columbia (15). The CDC has also been investigating reports of children presenting with acute flaccid myelitis associated with EV-D68 infection. Investigations are still ongoing to determine the role, if any, of EV-D68 infection among cases of neurologic illness identified during the same period $(15,16)$.

In September 2014, some Canadian jurisdictions reported laboratory-confirmed EV-D68 cases, including an association with neurologic findings $(17,18)$. As of December 15, 2014, laboratory-confirmed cases of EV-D68, primarily among those 18 years of age and younger have been detected in all ten provinces and one territory in Canada. EV-D68 is not a nationally notifiable disease in Canada, and thus little is known of the scale of its impact as a seasonal respiratory virus. Heightened awareness among public health and clinicians following the U.S. clusters likely contributed to the initial detection of cases in Canada.

Given the emergence of cases in Canada, and the limited knowledge of the epidemiology and full spectrum of disease caused by EV-D68, the Public Health Agency of Canada (PHAC), in collaboration with participating provincial and territorial $(\mathrm{P} / \mathrm{T})$ public health organizations, initiated a time-limited EV-D68 Severe Outcomes Surveillance Pilot in September 2014. The purpose of this article is to provide an epidemiological summary of hospitalized pediatric cases from the emergent stages of the EV-D68 outbreak in Canada.

\section{Investigations}

In September 2014, all provincial health agencies were invited to participate in the EV-D68 Severe Outcomes Surveillance Pilot. The following P/T health agencies and health centres participated in the surveillance pilot: Alberta Health; British Columbia Centre for Disease Control (BCCDC); New Brunswick Department of Health; Yukon Health and Social Services; Northwest Territories Department of Health and Social Services; Nunavut Department of Health; and Ontario (Public Health Ontario (PHO); the Children's Hospital of Eastern Ontario (CHEO); and the Hamilton Regional Laboratory Medicine Program (HRLMP), serving Hamilton Health Sciences, St. Joseph's Healthcare Hamilton, and other community hospitals in South Central Ontario). Management of the pilot project and the epidemiological analysis were conducted by PHAC's Centre for Immunization and Respiratory Infectious Diseases. 


\section{Case definition}

Cases were defined as persons $\leq 18$ years of age who were hospitalized and tested positive for EV-D68 infection by polymerase chain reaction (PCR) testing and/or genetic sequencing of the virus using standard enterovirus genotyping methods $(19,20)$, or an EV-D68 specific real-time PCR $(21)$, from any clinical specimen collected in September 2014.

\section{Data collection}

The following information was requested for all cases in aggregate form or in a de-identified line list: specimen collection date; sex; age; admission to an intensive care unit (ICU); clinical presentation (e.g., respiratory illness and/or neurologic illness); other clinical information (asthma); and outcomes (e.g., survived/died). Data collection was performed, where possible, using an enhanced surveillance case reporting form (CRF) (available upon request).

\section{Data analysis}

Basic descriptive analyses were performed to generate an epidemiological summary of hospitalized EV-D68 cases. Differences between groups (males versus females, and $<5$ years of age versus 5-18 years) were tested using Chi-square statistic with the assumption that EV-D68 is expected to infect an equal rate of individuals (by sex and age) as the population distribution in the jurisdictions reporting the cases. Expected values were calculated using population estimates for 2014 from Statistics Canada (22).

\section{Assessment}

Between September 1 and 30, 2014, there were 268 cases reported from three provinces: 210 (78.4\%) from Ontario (PHO), CHEO, and HRLMP); 45 (16.8\%) from Alberta; and 13 (4.9\%) from British Columbia. New Brunswick, Northwest Territories, Nunavut and Yukon had no cases to report with specimen collection dates during the month of September 2014. Of note, public primary and secondary schools had been closed in British Columbia due to a teachers' strike, with schools only having resumed from summer recess on September 22, 2014 (18). Data collection was performed by Alberta Health and BCCDC using the enhanced CRF, by PHO using the PHO Laboratories General Test Requisition (23), and by CHEO and HRLMP by review of medical and laboratory records. Close to half of all cases (119 or 44.4\%) had EV-D68 positive specimens collected in week 38 (September 14-20, 2014), while $60(22.4 \%)$ and 45 (16.8\%) had positive specimens collected in weeks 39 (September 21-27) and 37 (September 7-13), respectively (Figure 1).

Figure 1: Hospitalized pediatric ( $\leq 18$ years) cases of EV-D68 by specimen collection week, EV-D68 Severe Outcomes Surveillance Pilot, Canada, September 2014

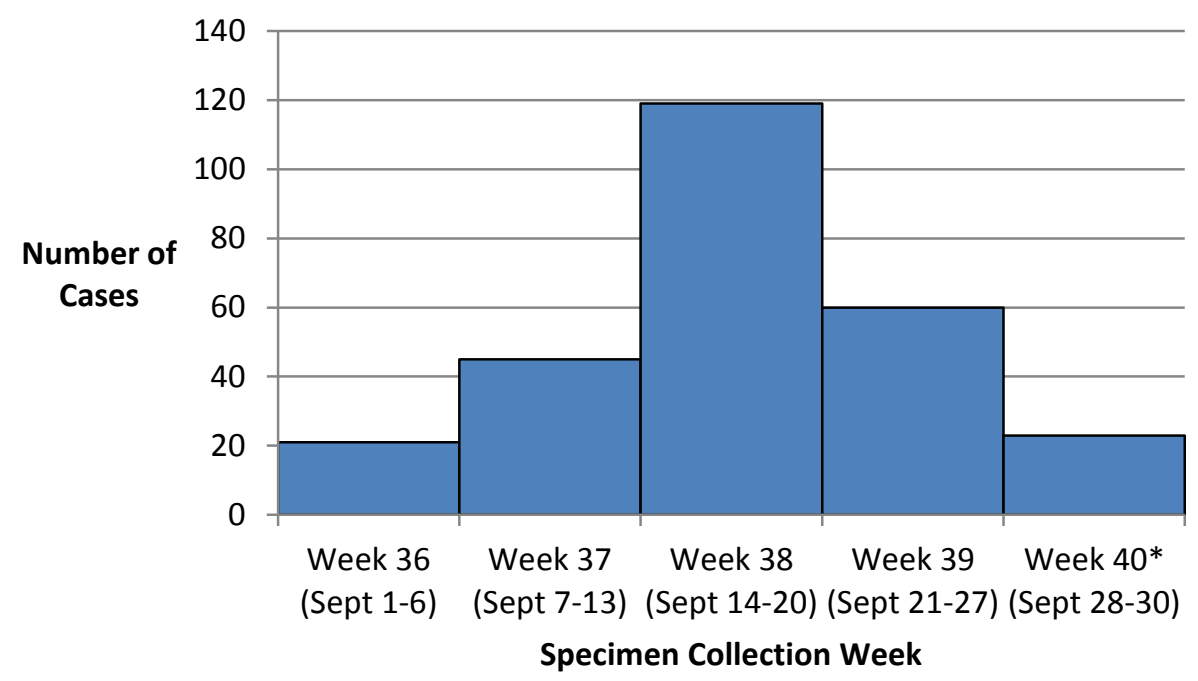

\footnotetext{
*Note: Week 40 does not include a full week of data. In addition, this epicurve represents a time-limited snapshot of hospitalized pediatric cases for September 2014, and may not be representative of the entire Canadian EV-D68 outbreak, continuing after this period.
} 


\section{Characteristics of cases}

Of the 268 cases with specimen collection dates in September 2014, 174 (64.9\%) were male; the sex difference was statistically significant $(p<0.01)$ (Table 1). Of the cases where age was available $(n=255)$, the mean age was 5.2 years ( 5.4 for males and 5.3 for females) and the median was 4.8 years ( 5.0 for males and 5.0 for females). The distribution of cases by age group is presented in the table. Half of the cases $(50.6 \%)$ were in children $<5$ years of age and roughly a third of the cases $(35.7 \%)$ were between the ages of 5 and 9 . In comparing the number of cases observed in young children ( $<5$ years) and those between 5 and 18 years of age, the difference was statistically significant $(p<0.01)$. The number of cases observed in young children $(n=129)$ was twice the number expected based on the population distribution.

Table 1: Descriptive epidemiological summary of hospitalized pediatric EV-D68 cases, EV-D68 Severe Outcomes Surveillance Pilot, Canada, September 2014

\begin{tabular}{|c|c|c|}
\hline ( $N=$ number for which information was available) & $\mathbf{n}$ & $\%$ \\
\hline \multicolumn{3}{|l|}{$\operatorname{Sex}(N=268)^{a}$} \\
\hline Male & 174 & 64.9 \\
\hline Female & 94 & 35.1 \\
\hline \multicolumn{3}{|l|}{ Age group $(\mathrm{N}=255)$} \\
\hline$<2$ years & 56 & 22.0 \\
\hline $2-4$ years & 73 & 28.6 \\
\hline $5-9$ years & 91 & 35.7 \\
\hline $10-14$ years & 31 & 12.1 \\
\hline $15-18$ years & 4 & $\overline{1.6}$ \\
\hline \multicolumn{3}{|l|}{ Admitted to intensive care unit $(\mathrm{N}=266)$} \\
\hline Yes & 18 & 6.8 \\
\hline No & 248 & 93.2 \\
\hline \multicolumn{3}{|l|}{ Clinical presentation $(\mathrm{N}=\mathbf{2 3 1})$} \\
\hline Respiratory IIIness only & 227 & 98.3 \\
\hline Neurologic Illness only & 1 & 0.4 \\
\hline Respiratory and neurologic illness & 2 & 0.9 \\
\hline No respiratory or neurologic illness & 1 & 0.4 \\
\hline
\end{tabular}

Of the 266 cases for whom ICU admission status was known, 18 (6.8\%) were admitted to an ICU. Of these 18 cases, $66.7 \%(n=12)$ were male and the sex distribution was similar to the distribution for all cases. The mean (5.5) and median (5.6) ages for ICU-admitted cases were slightly higher than the mean and median for all cases. Compared to the mean and median age for all cases by sex, these values were higher for males admitted to ICU (mean=6.4, median=6.0) and lower for females admitted to ICU (mean=4.0, median=3.6). Of the cases with clinical presentation data available $(n=231)$, respiratory illness was present in 229 cases $(99.1 \%)$, and neurologic illness was present in 3 cases (1.3\%). Of these three cases, one presented with seizures, one with symptoms of acute flaccid paralysis, and the other with unspecified neurologic symptoms. There was one case that presented with neither respiratory nor neurological symptoms. Of the 90 cases with additional clinical information available, $39(43.3 \%)$ were reported as having asthma. No deaths were reported among the 268 cases. 


\section{Conclusion}

Outbreaks of EV-D68 had not been detected in Canada prior to 2014. Initial outbreaks of an emerging pathogen are typically first recognized on the basis of their most severe presentations and can skew the impression of overall severity associated with that pathogen. Enhanced surveillance on a population level expands the understanding of the spectrum of illness to include milder presentations, even among hospitalized cases.

Whereas a very high proportion of pediatric cases required intensive care in the initial hospital clusters detected in Kansas City (100\%) and Chicago (91\%) in August 2014 (13), this represents only a subset of all cases that likely occurred within the geographic catchment area of those hospitals. Similarly, our enhanced surveillance, although conducted across seven provinces and three territories, includes only laboratory-confirmed cases of EV-D68 that were hospitalized. Looking at these hospitalized cases, we report here a median age (4.8 years) consistent with what was seen initially in Kansas City (4 years) and Chicago (5 years) (13). The interesting male preponderance we report is also consistent with other surveillance summaries of enterovirus-infected persons elsewhere (9). As with other enteroviruses, it is likely that a large proportion of milder community cases occurred for which no medical visit or diagnostic testing was sought or required at all, and these mild presentations and their accompanying epidemiologic characteristics will be missed almost entirely in most surveillance summaries.

While the majority of cases observed since September 2014 have been among those 18 years of age and younger, there have also been a small number of adult cases reported $(5,17,18)$, but are not included here It was decided that to provide an early snapshot of the outbreak, and for consistency across participating jurisdictions, this epidemiological summary would be restricted to pediatric cases from September 1 to 30, 2014, only. The decision to focus on pediatric populations was supported by the growing body of evidence showing that EV-D68 disproportionately affects younger populations compared to adults $(6-8,10-13,16,24-27)$. Cases in Canada have been observed beyond September, and in British Columbia, where a teachers' strike delayed the autumn return to school, a later seasonal peak in EV-D68 was observed. Therefore, ongoing surveillance in pediatric and adult populations will be important to develop a fuller understanding of disease seasonality, age-related susceptibility, and severity.

There are limitations to these data. This epidemiological summary is focused on a specific population (high-risk) and a period early in the outbreak, and may not be representative of the full EV-D68 outbreak in Canada in 2014. In addition, for those cases for which ICU admission was reported, it is unknown the extent to which EV-D68 infection contributed to the ICU admission. Also, jurisdictions conducted laboratory testing for EV-D68 using different methods that inherently provide varying levels of sensitivity and specificity $(19,28)$. Given that EV-D68 is not a notifiable disease nationally or within provinces and territories, some jurisdictions faced early obstacles in conducting enhanced case follow-up and reporting, and differed in their approaches to data collection. This may explain a portion of the missing results in this report and likely resulted in cases that were missed for follow-up. Similarly, jurisdictions vary in their surge capacity to respond to unexpected and emerging public health events. The EV-D68 experience is a reminder that timely public health response requires supportive legislation and infrastructure to enable the investigation of emerging pathogens which, by their very nature, occur without advance notice and may not be specifically pre-named in notifiable disease lists.

The EV-D68 Severe Outcomes Surveillance Pilot represents the first attempt to describe the epidemiological characteristics of severe pediatric cases of EV-D68 at the national level in Canada, and provides valuable insights into the early stages of the 2014 outbreak. Limitations of these findings should be considered in any attempts to extrapolate to the "Canadian" experience or in comparisons with other jurisdictions. Continued investigation is warranted to more fully define the seasonality, susceptibility and spectrum of illness associated with this emerging pathogen and to develop appropriate public health measures to mitigate its impact. 


\section{Acknowledgements}

This report would not have been possible without the contributions of all federal, provincial and territorial health organizations and health centres that participated or contributed data for the Canadian EV-D68 Severe Outcomes Surveillance Pilot. In addition, we would like to acknowledge the following: Dr. Dana Paquette, Program Director, Canadian Field Epidemiology Program; Dr. Robert Stirling, Public Health Physician, Public Health Ontario; the Alberta Provincial Laboratory for Public Health staff for work on routine specimen testing; British Columbia would like to acknowledge the public health nurses and epidemiologists in regional Health Authorities who completed case report forms and compiled the enhanced surveillance information, with specific acknowledgement Christina Fung, Margot Smythe, Maritia Gully, Kelly Yu and Gillian Frosst; individuals from the New Brunswick Department of Health-Shelley Landsburg, Suzanne Savoie and Louis-Alexandre Jalbert; Vitalité Health Network with special collaboration of the microbiology laboratory of the George L. Dumont University Hospital Centre; Horizon Health Network; Yukon Health and Social Services; Northwest Territories Office of the Chief Public Health Officer; Department of Health, Government of Nunavut; Emily De Rubeis and Veeran-Anne Singh from the First Nations and Inuit Health Branch in Health Canada.

\section{Conflict of interest}

None

\section{Funding}

None

\section{References}

(1) International Committee on Taxonomy of Viruses, King AMQ. Virus taxonomy: Classification and nomenclature of viruses: Ninth report of the international committee on taxonomy of viruses. London; Waltham, MA: Academic Press; 2012:1327.

(2) Schieble JH, Fox VL, Lennette EH. A probable new human picornavirus associated with respiratory diseases. Am J Epidemiol. 1967;85(2):297-310.

(3) Oberste MS, Maher K, Schnurr D, et al. Enterovirus 68 is associated with respiratory illness and shares biological features with both the enteroviruses and the rhinoviruses. J Gen Virol. 2004; 85(Pt 9):2577-84. doi: 10.1099/vir.0.79925-0.

(4) Khetsuriani N, Lamonte-Fowlkes A, Oberst S, Pallansch MA, Centers for Disease Control and Prevention. Enterovirus surveillance-United States, 1970-2005. MMWR Surveill Summ. 2006; 55(8):1-20. doi: ss5508a1.

(5) National Collaborating Centre for Infectious Diseases. Disease debrief: EV-D68. Updated 2014 [cited 2014 Dec]. http://www.nccid.ca/disease-debrief-ev-d68.

(6) Imamura T, Fuji N, Suzuki A, et al. Enterovirus 68 among children with severe acute respiratory infection, the Philippines. Emerg Infect Dis. 2011; 17(8):1430-5. doi: 10.3201/eid1708.101328.

(7) Kaida A, Kubo H, Sekiguchi J, et al. Enterovirus 68 in children with acute respiratory tract infections, Osaka, Japan. Emerg Infect Dis. 2011; 17(8):1494-7. doi: 10.3201/eid1708.110028.

(8) Ikeda T, Mizuta K, Abiko C, et al. Acute respiratory infections due to enterovirus 68 in Yamagata, Japan between 2005 and 2010. Microbiol Immunol. 2012;56(2):139-43. doi: 10.1111/j.1348-0421.2012.00411.x.

(9) Meijer A, Benschop KS, Donker GA, van der Avoort HG. Continued seasonal circulation of enterovirus D68 in the Netherlands, 2011-2014. Eurosurveillance. 2014; 19(42).

http://www.eurosurveillance.org/ViewArticle.aspx?Articleld=20935

(10) Meijer A, van der Sanden S, Snijders BE, et al. Emergence and epidemic occurrence of enterovirus 68 respiratory infections in the Netherlands in 2010. Virology. 2012; 423(1):49-57. doi: 10.1016/j.virol.2011.11.021.

(11) Jacobson LM, Redd JT, Schneider E, et al. Outbreak of lower respiratory tract illness associated with human enterovirus 68 among American Indian children. Pediatr Infect Dis J. 2012;31(3):309-12. doi: 10.1097/INF.0b013e3182443eaf.

(12) Centers for Disease Control and Prevention (CDC). Clusters of acute respiratory illness associated with human enterovirus 68-Asia, Europe, and United States, 2008-2010. MMWR. 2011; 60(38):1301-4. doi: mm6038a1.

(13) Midgley CM, Midgley CM, Selvarangan R, et al. Severe respiratory illness associated with enterovirus D68-Missouri and Illinois, 2014. MMWR. 2014; 63(36):798-9.

(14) Hasegawa S, Hirano R, Okamoto-Nakagawa R, Ichiyama T, Shirabe K. Enterovirus 68 infection in children with asthma attacks: Virus-induced asthma in Japanese children. Allergy. 2011; 66(12):1618-20. doi: 10.1111/j.13989995.2011.02725.x.

(15) Human enterovirus D68 North America (20): Update. ProMED. 2014 Dec 15. Archive No. 20141215.3035008. 
http://www.promedmail.org/direct.php?id=3035008

(16) Pastula DM, Aliabadi N, Haynes AK, et al. Acute neurologic illness of unknown etiology in children-Colorado, August-September 2014. MMWR. 2014; 63(40):901-2. doi: mm6340a5.

(17) Fonseca K, Kellner JD., Talbot J, et al. Human enterovirus 68-Canada: (Alberta). ProMED. 2014 Sep 16 [cited 2014 Dec]. Archive No. 20140916.2775534.

http://www.promedmail.org/direct.php?id=2775534

(18) Murti M, Pollock S, Krajden M, Tang P, Chambers C, Skowronski D. Polio-like illness-North America: Canada (British Columbia). 2014 Sep 30 [cited 2014 Dec 29]. http://www.promedmail.org/direct.php?id=2819618

(19) Nix WA, Oberste MS, Pallansch MA. Sensitive, seminested PCR amplification of VP1 sequences for direct identification of all enterovirus serotypes from original clinical specimens. J Clin Microbiol. 2006;44(8):2698-704. doi: 44/8/2698.

(20) Rahamat-Langendoen J, Riezebos-Brilman A, Borger R, et al. Upsurge of human enterovirus 68 infections in patients with severe respiratory tract infections. J Clin Virol. 2011; 52(2):103-6. doi: 10.1016/j.jcv.2011.06.019.

(21) Centers for Disease Control and Prevention (CDC). Enterovirus D68 (EV-D68) 2014 outbreak strain-specific real-time reverse transcription / polymerase chain reaction (rRT-PCR) assay instructions-Version 10/14/2014 [cited 2014 Dec 30]. http://www.cdc.gov/non-polio-enterovirus/downloads/EV-D68-RT-PCR-protocol.pdf

(22) Statistics Canada. Table 051-0001-Estimates of population, by age group and sex for July 1, Canada, Provinces and Territories, annual (persons unless otherwise noted). CANSIM.

(23) Public Health Ontario. General test requisition [cited 2014 Dec 23]. http://www.publichealthontario.ca/en/eRepository/General_test_fillable_requisition.pdf

(24) Enterovirus targets children in central United States. Medical Läboratory Observer. 2014; 46(10):6.

(25) Baum SG. Severe respiratory illness associated with EV-D68. NEJM Journal Watch. Infectious Diseases. 2014:n/a. doi: http://dx.doi.org/10.1056/nejm-jw.NA35680.

(26) Dietz V, Andrus J, Olive JM, Cochi S, de Quadros C. Epidemiology and clinical characteristics of acute flaccid paralysis associated with non-polio enterovirus isolation: The experience in the Americas. Bull World Health Organ. 1995; 73(5):597-603.

(27) Renois F, Bouin A, Andreoletti L. Enterovirus 68 in pediatric patients hospitalized for acute airway diseases. J Clin Microbiol. 2013; 51(2):640-3. doi: 10.1128/JCM.02640-12.

(28) Oberste MS, Penaranda S, Rogers SL, Henderson E, Nix WA. Comparative evaluation of taqman real-time PCR and semi-nested VP1 PCR for detection of enteroviruses in clinical specimens. J Clin Virol. 2010;49(1):73-74. doi: 10.1016/j.jcv.2010.06.022. 